EPJ Web of Conferences 92,02092 (2015)

DOI: $10.1051 /$ epjconf/ 20159202092

C Owned by the authors, published by EDP Sciences, 2015

\title{
Drag force and jet propulsion investigation of a swimming squid
}

\author{
Mahdi Tabatabaei ${ }^{1}$, Ali Bahadır Olcay ${ }^{2, a}$, Gökhan Gokçen ${ }^{3}$ and Hasan A. Heperkan ${ }^{1}$ \\ ${ }^{I}$ Yildiz Technical University, Faculty of Mechanical Engineering, Besiktas, Istanbul, 34349, Turkey \\ ${ }^{2}$ Yeditepe University, Mechanical Engineering Department, Atasehir, Istanbul, 34755, Turkey \\ ${ }^{3}$ Dogus University, Mechanical Engineering Department, Kadikoy, Istanbul, 34722, Turkey
}

\begin{abstract}
In this study, CAD model of a squid was obtained by taking computer tomography images of a real squid. The model later placed into a computational domain to calculate drag force and performance of jet propulsion. The drag study was performed on the CAD model so that drag force subjected to real squid was revealed at squid's different swimming speeds and comparison has been made with other underwater creatures (e.g., a dolphin, sea lion and penguin). The drag coefficient (referenced to total wetted surface area) of squid is 0.0042 at Reynolds number $1.6 \times 106$ that is a $\% 4.5$ difference from Gentoo penguin. Besides, jet flow of squid was simulated to observe the flow region generated in the $2 \mathrm{D}$ domain utilizing dynamic mesh method to mimic the movement of squid's mantle cavity.
\end{abstract}

\section{Introduction}

Swimming techniques of marine animals for developing new technologies have been attracted many engineers and researchers. The shape of modern submarines and ship are an example that engineer applies for design. One of the marine animals that are studied by researchers is a squid. Squid can move very fast under water and control its propulsion by providing high velocity in a short time period and obtain high swimming performance. In this study, we focus on characteristics of the squid hydrodynamic drag and jet system because jet propulsion appears to be providing high forward speeds. A streamlined body shape is most important to reduce drag in a high viscosity environment and swimming performance in under water animals [1-2]. Relation between Reynolds number and drag coefficient in marine animals was studied experimentally by Stelle [3]. He measured drag coefficient and boundary layer thickness in Sea Lion by using video analysis. Fish [4] defined swimming of dolphins at different velocities in large pools. The study includes coefficient of drag and lift in different angle of attack and the maximum angle of attack causes a linear decrease with velocity. Locomotion of squid was determined by O'Dor [5]. He applied video model for calculating drag coefficient and lift coefficient in different speeds and mantle diameters because squid mantle diameter change when it accelerates forward. Rahman et. al. [6] studied flow computation around the swimming motion of a squid-like robot with two undulating side fins, mimicking those of a Stingray or a Cuttlefish. They did numerical simulations for various aspect ratios, fin angles and frequencies in order to validate the proposed relationship among principal dimensions. They discussed flow characteristics and hydrodynamic forces acting on the body and fin. They established a simple relationship among the fin's principal dimensions. $\mathrm{Yu}$ et al. [7] numerically investigated a fish's mechanical capability and suitable timing to execute maneuvers from a steady straight-line swimming state. They calculated longitudinal forces and yaw moment acting on a fish for varied slip number. They concluded that decreasing slip number increases mechanical capability for a fish to execute both longitudinal and sideway maneuvers because the amplitudes of both net longitudinal force coefficient and net yaw moment coefficient are enhanced. Yi-gang and De-cheng [8] investigated numerically the self-propelled motion of a fish with a pair of rigid pectoral fins. They developed a Navier-Stokes equation solver incorporating with the multi-block and overset grid method to deal with the multi-body and moving body problems. It is concluded that the fin can generate great thrust and at the same time have no generation of lift force for the liftswimming mode. On the other hand they investigated unsteady flow features such as periodic vortex structure generation and shedding. Eloy [9] evaluated the swimming performances of aquatic animals using the dimensionless quantity Strouhal number. Experimental observations showed that flapping foils had maximum propulsive efficiency in the interval $0.25<\mathrm{St}<0.35$. Polidori et al [10] studied determining skin-friction drag analysis in underwater swimming quantifying the effect of the temperature gradient between swimmer's body and

\footnotetext{
${ }^{a}$ Corresponding author: bahadir.olcay@yeditepe.edu.tr
} 
pool water. In steady flow conditions, they determined surface shear stresses and the skin-friction drags. They concluded that the skin-friction drag decreased 5.3\%, independently from swimming speeds, with increasing average boundary-layer temperature provided that the flow remained laminar. Loebbecke et al [11] investigated olympic level athletes swimming underwater using the dolphin kick. Swimming velocity is varied in the range of 1.12 and 1.85 which corresponded to a range of effort levels. They measured body length, time taken by the swimmer to traverse a body length, kick amplitude at the toes. Using these parameters average velocity of the swimmer, kick frequency, the reduced or length-specific velocity and the non-dimensional quantities kick amplitude were determined. They concluded that human and cataceans had comparable non-dimensional kick amplitudes, but kick frequency in humans was greater than for cetaceans swimming at equivalent speeds. Bartol and Krueger [12] investigated jet propulsion of squids by using experimental methods. They used DPIV technique to obtain velocity and vorticity vector fields of the domain and they explained relation between jet angel and speed. Anderson and Grosenbaugh [13] perused squid locomotion that includes jet propulsion and fin. He exposed effect of relative between fin and jet propulsion. Squid can change fin shape at high speeds and the fins use to control its dynamic balance. Characteristics of vortex ring formation were studied by some researchers experimentally and numerically [14-16]. They used cylinder and piston mechanism for ejecting water into motionless water to explain the formation of vortex rings in a jet flow. Behavior of vortex ring is different and it is highly depend on $\mathrm{L} / \mathrm{D}$. When $\mathrm{L} / \mathrm{D}$ was greater than about 3.6-4.5, a trail of vorticity followed head vortex ring [15]. Bera [17] studied a purely alternating jet with zero massflux and a mixed pulsed jet with an additional blowing component via particle image velocimetry (PIV). The jets were emanated from a two-dimensional slit connected to a converging nozzle opening normally from a flat wall. The pulsatile motion of the jet was achieved by a loudspeaker. It was stated that a large lateral expansion of the jet and a large entrainment rate of external fluid occurred for unsteady jets, compared to usual steady jets. They concluded that in the case of purely alternating jet, the expansion took place close to the slit, on the other hand in the case of mixed pulsed jet the vortices developed farther from the orifice. Jing-lei [18] experimentally investigated the instantaneous flow characteristics of a circular orifice synthetic jet, including the forming, developing and breaking down of the vortex of the jet via phase-locked Particle Image Velocimetry method. They changed orifice depths from $1.5 \mathrm{~mm}$ to 2 $\mathrm{mm}$ and $3.5 \mathrm{~mm}$ in order to study the effect of orifice depth on the flow structure. It was concluded that the peak of the mass flux and momentum flux increased as the orifice depth increased. Carpy and Manceau [19] studied the ability of different turbulence models to close the phase-averaged Navier-Stokes equations of statistically periodic Synthetic jet flows. They compared their results with literature. They showed that the evolution of the vortex dipole generated by inviscid mechanisms was essentially inviscid during the early blowing phase, when the flow was more transitional than fully turbulent. Bremhorst and Gehrke [20] investigated fully pulsed air jet exhausting into still air examining turbulent kinetic energy budges in the jet flow. Measurements were conducted via hot-wire anemometers. Quasi-steady jet was achieved by pulsing with very low Strouhal numbers. They concluded that axial diffusion of turbulent energy varied strongly with distance but radial diffusion was almost self-similar for the region investigated. Kim et al [21] examined the twotime-scale irrotational-strain-sensitized turbulence model in literature comparing experimental data for steady and fully-pulsed turbulent round jets. The fully-pulsed turbulent round jets was simulated for over a range of frequencies from 2 to $10 \mathrm{~Hz}$. Xiaoyong et al [22] examined and compared two cases with different simulation methods in low Reynolds number laminar flow. They used periodic velocity inlet and dynamic mesh boundary conditions to simulate oscillatory piston of synthetic jet. They revealed that the vortex dyestructure was almost same outside of cave and the velocity profiles near the orifice were different distinctly on $0^{\circ}$ and $180^{\circ}$ phase, the max speed difference at center of orifice was about $0.017 \mathrm{~m} / \mathrm{s}$ at the end of third period, which increased by $150 \%$ of the simulating results with dynamic mesh boundary method. At least they concluded that the simulation method with dynamic mesh was more reasonable because it was realistic motion of piston. Hsua et al. [23] conducted experiments to compare flow fields of non-zero-net-mass-flux double-acting synthetic jets and single-acting synthetic jets was performed with water as the working fluid. The actuation frequencies were changed in the range of $2 \mathrm{~Hz}$ to $12 \mathrm{~Hz}$. They concluded that double-acting synthetic jets revealed good potential with significant vorticity enhancement for the design of synthetic jet in heat transfer applications. Yang et al [24] presented a study to illustrate a framework for combining Digital Particle Image Velocimetry measurements with Lagrangian analysis tools that analyze the starting vortex ring generated by a thin circular disk. They showed that the existence of a flux window between repelling Lagrangian Coherent Structures and attracting Lagrangian Coherent Structures, though that the shear flow was entrained into vortex. The results showed that the vortex formation process was completed at time $\mathrm{t}=2714 \mathrm{~ms}$. We investigate the real squid hydrodynamic drag and jet flow system in different Reynolds number of external and internal flow.

\section{Material and Methodology}

\subsection{Computer tomography images of a real squid}

8 samples are scanned using computed tomography. From the preliminary scans it was known that the dead squids flatten on a hard surface. To prevent flattening one method was to fill the body of the squid with silicone gel. First group is scanned on paper bedding without silicone gel injection. The second group is scanned on a paper bedding with silicone gel injection. And the last group is 
scanned while hanged and filled with silicone gel whereas one of them was not filled with silicone gel. Best results are obtained from the scans of the squid samples with silicone gel injection and hanged. The study continued with the selected scan from this group. The results of scans are an image set in dicom format, figure 1. The set of 360 images presenting layer by layer x-ray scans, are used to construct a surface in stl format using Amira v5.5 Software (Visualization Sciences Group, SAS., Oregon) (Visualization Sciences Group, 2013. The first step of this construction is to increase the contrast of the images in order to distinguish the soft tissue from the surroundings. In order to include the legs and the tentacles of the squid in the numerical model a detailed segmentation is needed. One problem is that although the legs and the tentacles of the hanged dead animal is similar to the shape of the swimming squid, there where irregular voids, figure 2 . The filling and smoothing of the leg structure should be done layer by layer at every image. At the same time the inside of the body is filled, figure 3 .

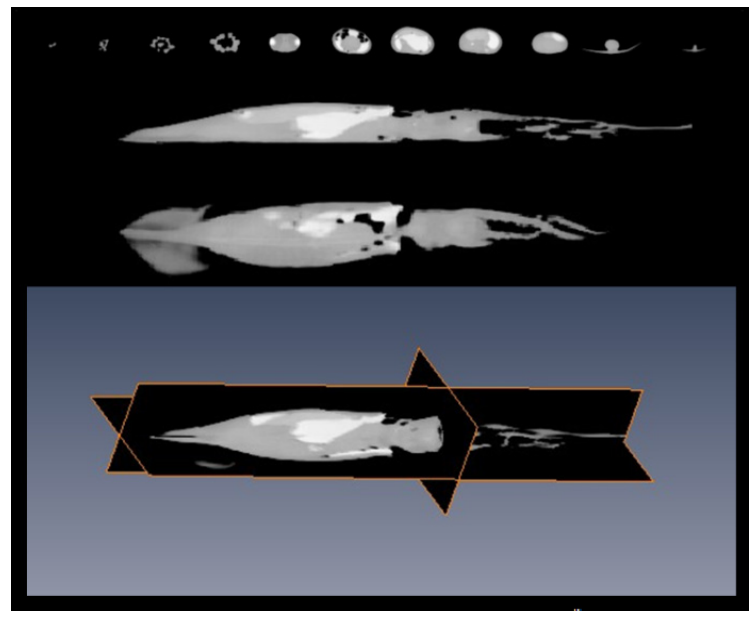

Figure 1. Dicom images obtained by ct scan. Above (30.-60.90.-120.-150.-180.-210.-240.-270.-300. ad 330

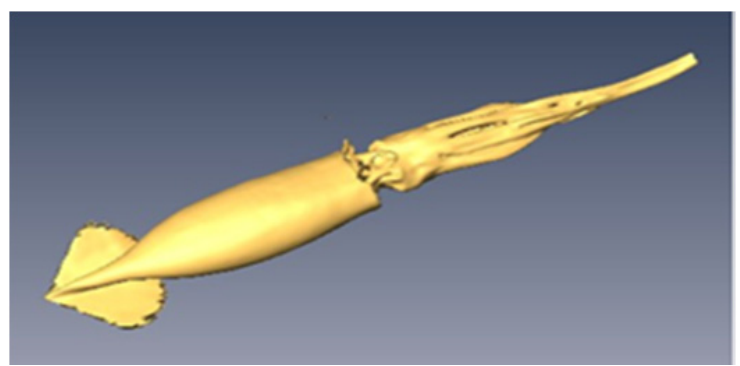

Figure 2. Surface obtained after scan.

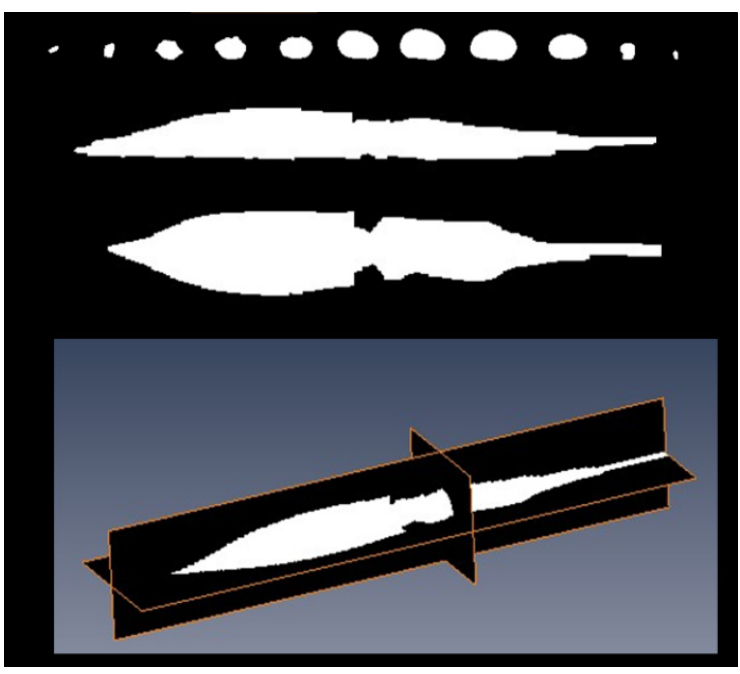

Figure 3. Surface obtained after segmentation. Above (30.-60.90.-120.-150.-180.-210.-240.-270.-300. ad 330. layers) below: side view below top view bottom composite view.

With the help of the Amira software and the segmentation done, the selected regions are interpolated to construct the three dimensional geometry. Figure 4a.The surface is exported to Meshlab Software (v1.3.1 Visual Computing Lab) [25] in stl format which expresses the surface that encompasses the geometry in triangles. Since the construction usually creates defects like double nodes and small voids it was searched for these kinds of defects and the found defects are fixed. Using the quadratic edge collapse decimation algorithm provided by the software surface is improved and expressed with less triangles without a loss is quality. The last step is smoothing of the surface, figure $4 \mathrm{~b}$.

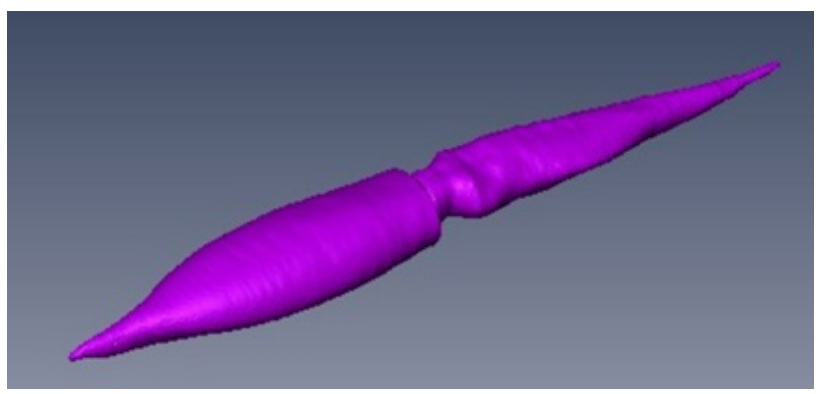

(a)

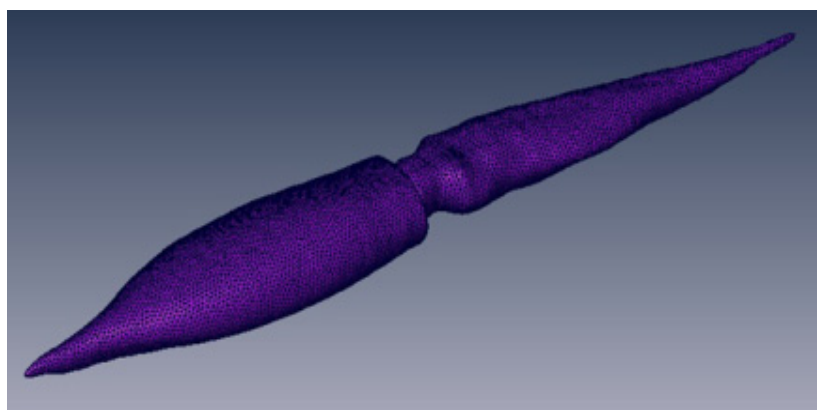

(b)

Figure 4. a (above) Surface model after segmentation $b$. (below) surface model after defect cleaning and smoothing. 
After cleaning, remeshing with less surface elements and smoothing the surface model is transferred to Rhinoceros v4.0 Software [26] in order to convert the model to a solid model (i.e. in stp format) which is required by the finite element analysis package ANSYS (Ansys Inc.). Finally the meshing is done outside the squid for drag and lift analyses. For the momentum analyses inside of the squid and domain behind of the squid is meshed in figure 5 .

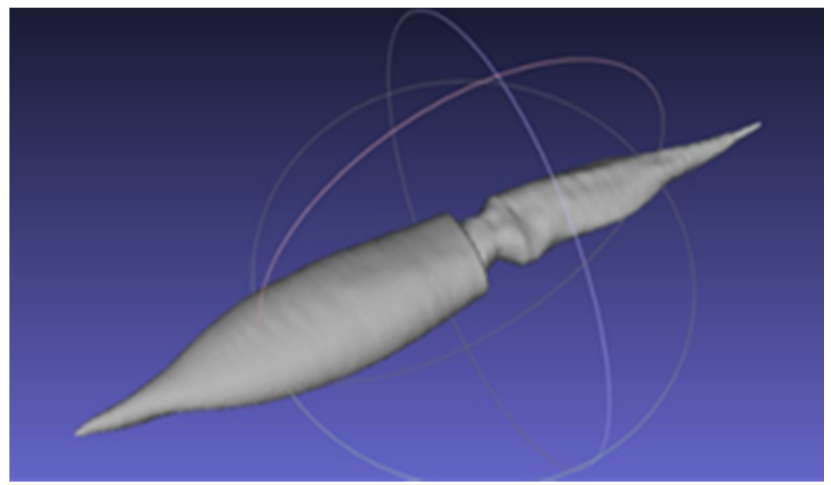

(a)

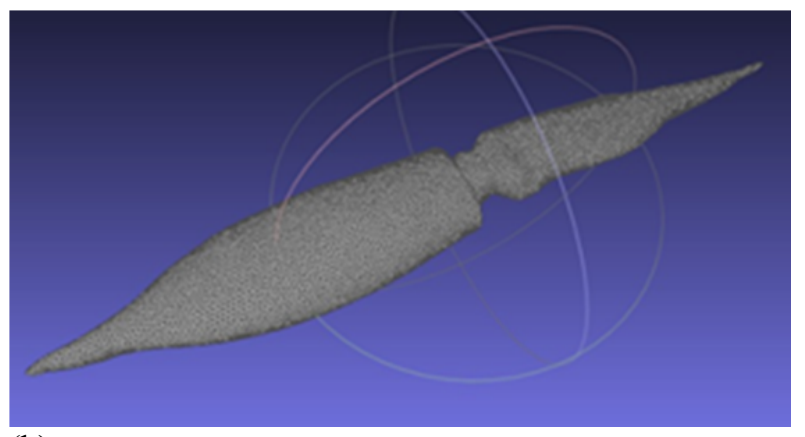

(b)

Figure 5. Meshed finite element model.

\subsection{Hydrodynamic drag}

In this study, real squid was modeled by computer tomography images for numerical simulation. A 2-D geometry was generated for analysis of drag force in different Reynolds numbers as illustrated in figure 6 . The equation describing Reynolds numbers is given by

$$
\operatorname{Re}=\rho \mathrm{UL} / \mu
$$

where $\mathrm{U}$ is the flow velocity, $\mathrm{L}$ is the characteristic linear (e.g., body length), $\rho$ and $\mu$ is the density and absolute viscosity of the fluid, respectively.

The equation describing total body drag is given by

$$
\text { Total Drag }=\frac{1}{2} \rho V^{2} C_{d}
$$

where $\rho$ is the density of the fluid, $\mathrm{V}$ is the velocity of the fluid relative to the body, $\mathrm{A}$ is the characteristic area of the body, and $\mathrm{Cd}$ is the drag coefficient. Two primary types of drag were investigated for this study: Firstly, skin friction drag, a tangential force, and results from shear stresses in the water sliding by the body. Secondly, pressure drag which is a perpendicular force on the body associated with the pressure difference between the front and back of the body. As body becomes more streamlined, the pressure drag becomes lower causing total body drag of a swimmer smaller. The fineness ratio of a squid, defined as maximum body length / maximum body diameter, in this study is 7.58. The geometry of domain is axisymmetric; thus, $\mathrm{x}$ coordinate is selected to be axis while $r$ coordinate is chosen as radial direction. The domain area is $150 \mathrm{D}$ in length and $9 \mathrm{D}$ in height. A total $1,715,018$ tet and quad elements with increased mesh density near the squid body were used for computation as shown in figure 7 and figure 8. Governing equations for the flow field was solved by a commercial CFD package, namely ANSYS Fluent.

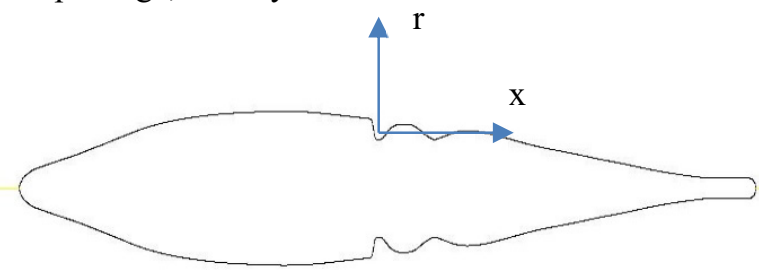

Figure 6. Axisymmetric geometry of squid.

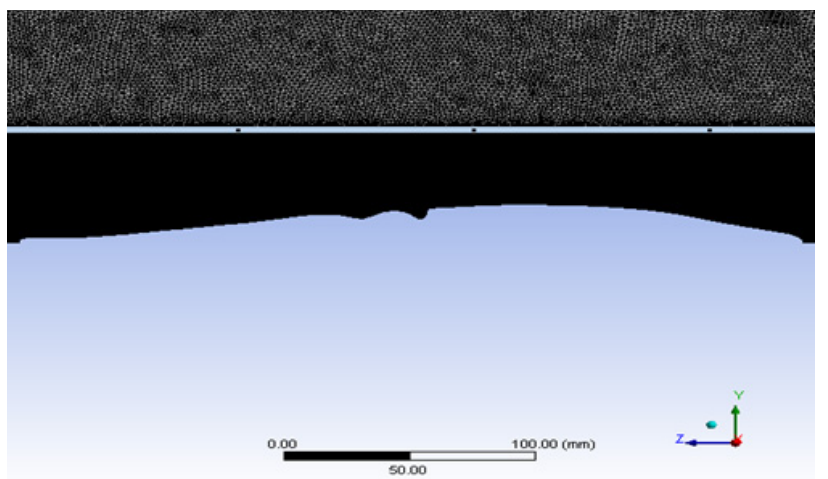

Figure 7. Meshing of domain for solving.

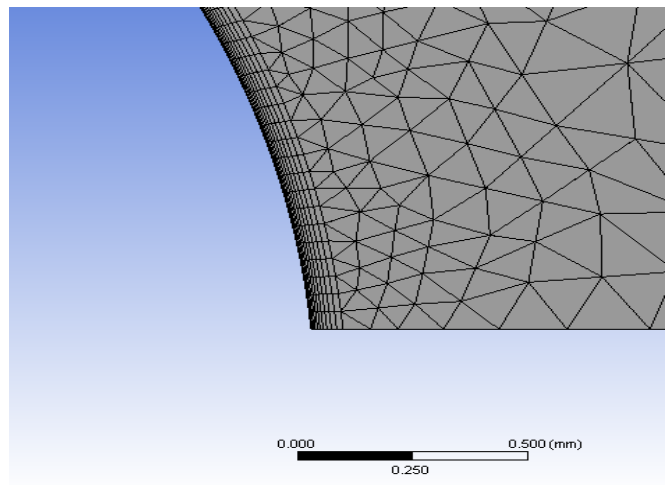

Figure 8. Enlarged mesh on squid surface.

\subsection{Jet propulsion}

A squid contracts its mantle cavity wall in order to pressurize the enclosed water in the mantle cavity prior to ejection of water. Once the squid starts to eject this 
pressurized water, mantle cavity wall must contract to provide required pressure for the jet flow. In this study, we investigated squid locomotion by using numerical methods by a commercial CFD package namely AnsysFluent. The geometry of domain is axisymmetric thus plane of axial ( $\mathrm{x}$ ) and radial $(\mathrm{r})$ direction is shown in Figure 9. The domain area is $300 \mathrm{D}$ in length and $40 \mathrm{D}$ in height while $26 \mathrm{D}$ is the length of mantle wall, $6 \mathrm{D}$ is the nozzle length and $6 \mathrm{D}$ is nozzle inlet diameter where $\mathrm{D}$ is the jet exit diameter. Squid's mantle cavity wall was moved during jet ejection by mimicking mantle cavity contraction. Geometry of squid's mantle cavity wall and domain used for jet propulsion analysis are shown in Figure 9a. Dynamic mesh method was utilized to move squid's mantle cavity walls. In this method, mantle cavity wall diameter decreases from $13 \mathrm{~cm}$ to $4 \mathrm{~cm}$ at 1 second time period. This wall movement is programmed such that wall actually follows a periodic sine velocity profile as shown in Figure 10a thru 10f. Movement of squid's mantle wall forces the fluid to accelerate passing through nozzle (enlarged view is given in Figure 9b). Once the fluid passes thru the nozzle exit plane, it enters to the solution domain where impulse and kinetic energy calculations are performed.

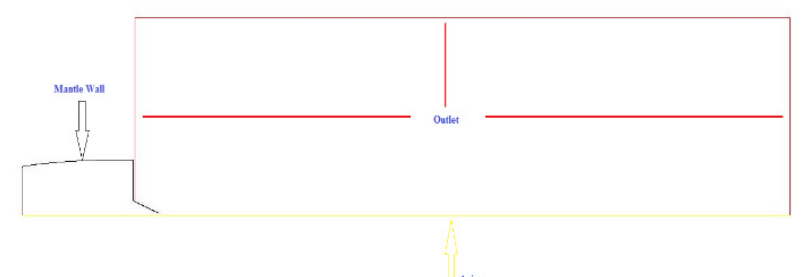

Figure 9a. Analysis domain of jet flow and squid mantle simulation.

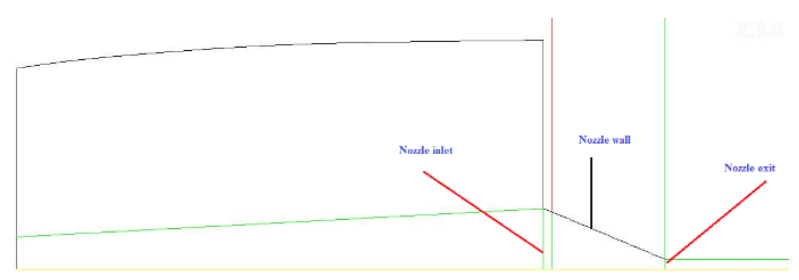

Figure 9b. Enlarged view of nozzle.

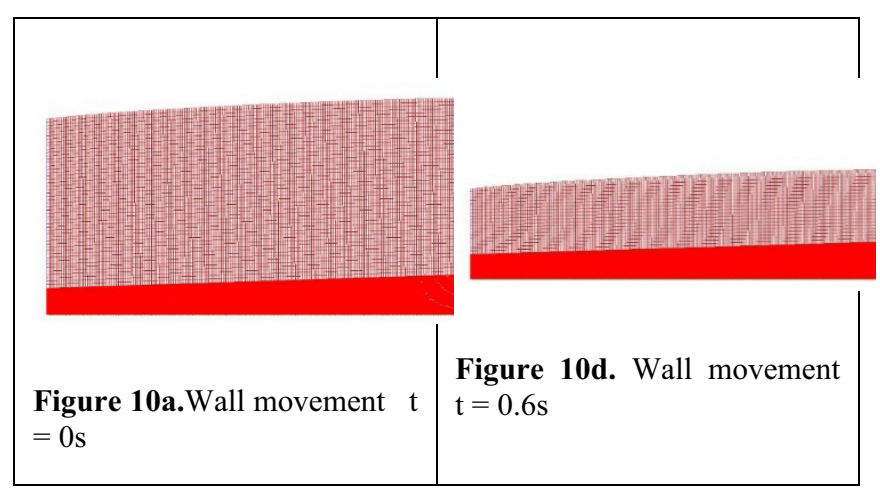

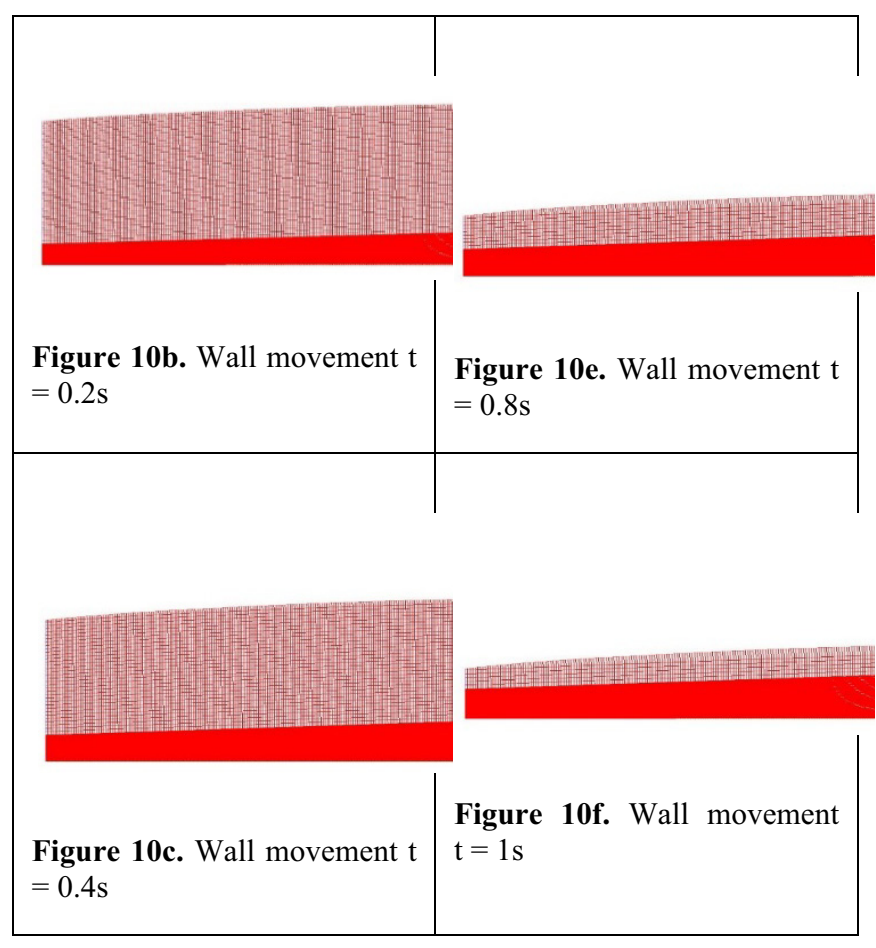

\section{Results}

\subsection{Drag coefficient}

Drag coefficient was calculated from $3.21 \mathrm{~m} / \mathrm{s}$ to 9.23 $\mathrm{m} / \mathrm{s}$ swimming speeds range and referenced to the animal's total wetted surface area $\left(C_{d, A}\right)$. Drag coefficient was compared for the squid with values obtained for other marine animals for three different Reynolds numbers as given in table 1 and figure 11 . The numerical results that were obtained for squid are in good agreement when they were compared by experimental results of sea lion, penguin and dolphin (Feldkamp 1987, Williams 1985, Videler 1985). The results show that drag coefficient decreases with growing Reynolds numbers. Figure 12 shows pressure distribution around squid body and a high pressure region around head of the squid. This causes resisting force to its forward motion in the water. Velocity contours of hydrodynamic boundary layer is shown in figure 13 No slip boundary condition cause zero velocity on body surface of squid and generate a boundary layer on surface of squid.

Table 1. Comparison of drag coefficient.

\begin{tabular}{|l|l|l|l|}
\hline & $\begin{array}{l}\mathrm{Re}=1,000, \\
000\end{array}$ & $\mathrm{Re}=1,600,000$ & $\begin{array}{l}\mathrm{Re}=2,870, \\
000\end{array}$ \\
\hline $\begin{array}{l}\text { Drag coefficient- } \\
\text { Squid*(present } \\
\text { study) }\end{array}$ & 0.00448 & 0.0042 & 0.003975 \\
\hline $\begin{array}{l}\text { Drag } \\
\text { coefficient- } \\
\text { Gentoo } \\
\text { penguin }\end{array}$ & 0.0044 & - & - \\
\hline
\end{tabular}




\begin{tabular}{|l|l|l|l|}
\hline $\begin{array}{l}\text { Drag } \\
\text { coefficient- } \\
\text { estuary dolphin }\end{array}$ & 0.004 & - & - \\
\hline $\begin{array}{l}\text { Drag } \\
\text { coefficient- } \\
\text { Harbor seal }\end{array}$ & - & 0.004 & - \\
\hline $\begin{array}{l}\text { Drag } \\
\text { coefficient- } \\
\text { Sea lion }\end{array}$ & - & - & 0.0039 \\
\hline
\end{tabular}

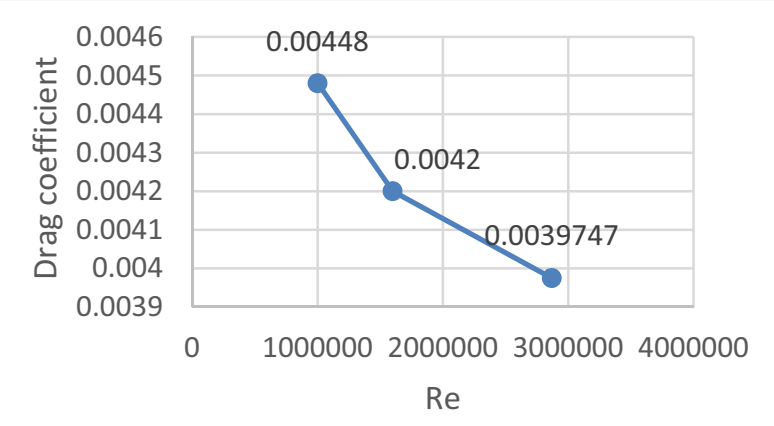

Figure 11. Drag coefficient decrease with growing Reynolds numbers.

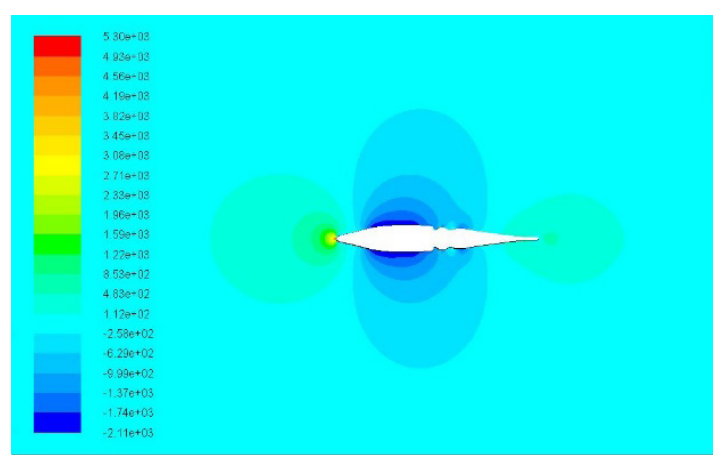

Figure 12. Pressure distribution around squid body.

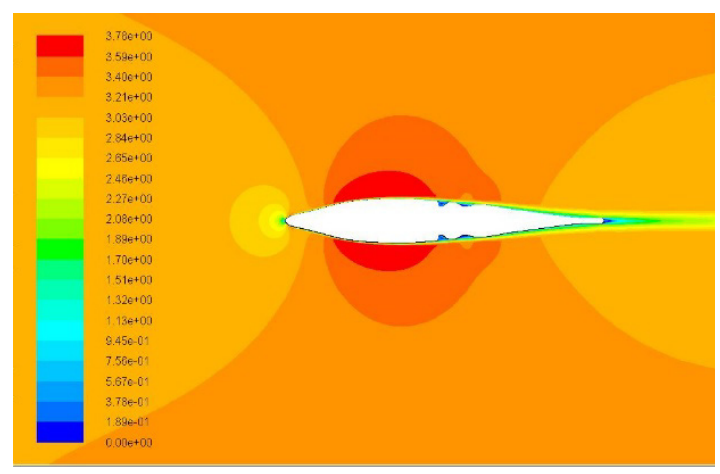

Figure 13. Contours of velocity.

\subsection{Jet propulsion}

In this study, we investigated jet flow that was generated by squid in $1 \mathrm{~s}$ periodic time. The behaviors of velocity magnitude in various time shown figure 14a. thru 14f. By moving mantle cavity wall of squid the fluid flows at nozzle and jet flow starts to generate in the nozzle then develop on fluid field.

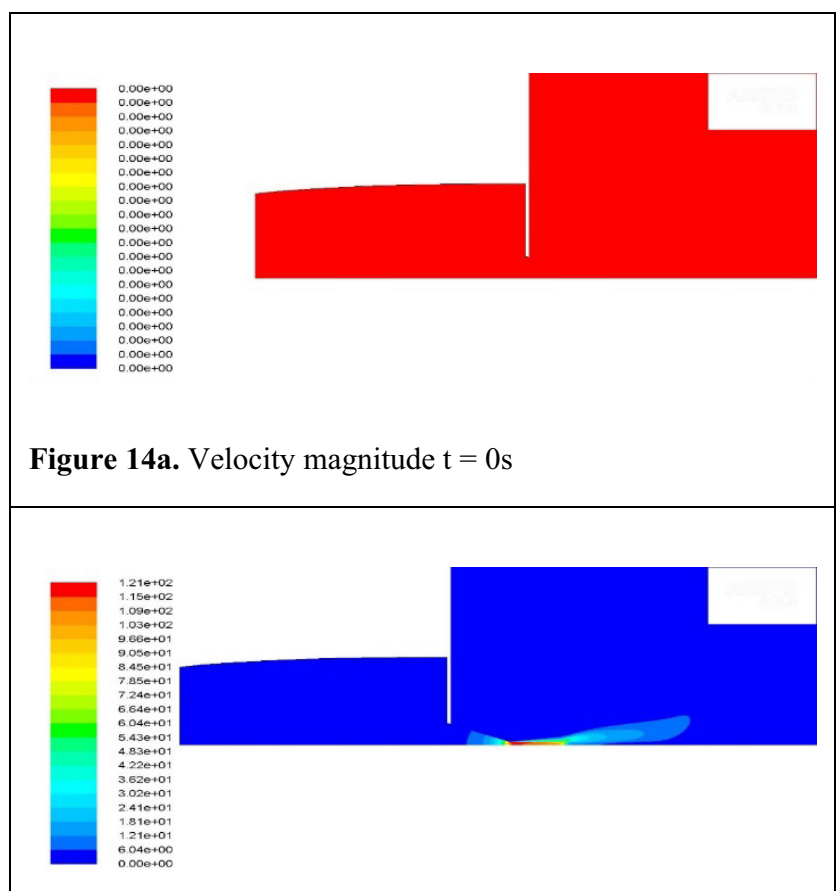

Figure 14b. Velocity magnitude $t=0.2 \mathrm{~s}$

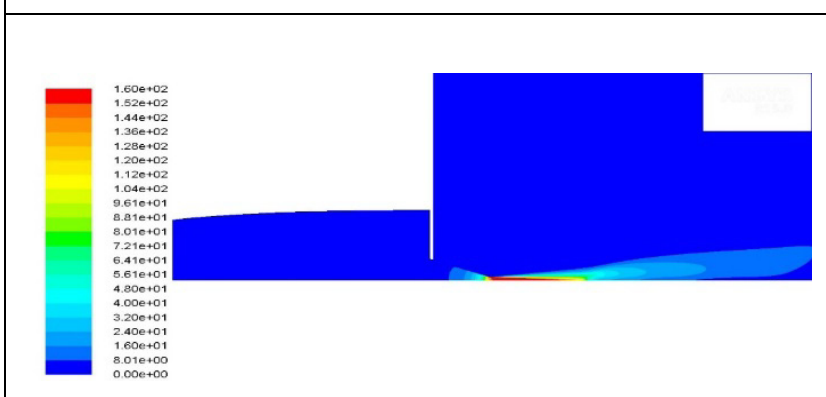

Figure 14c. Velocity magnitude $t=0.4 \mathrm{~s}$

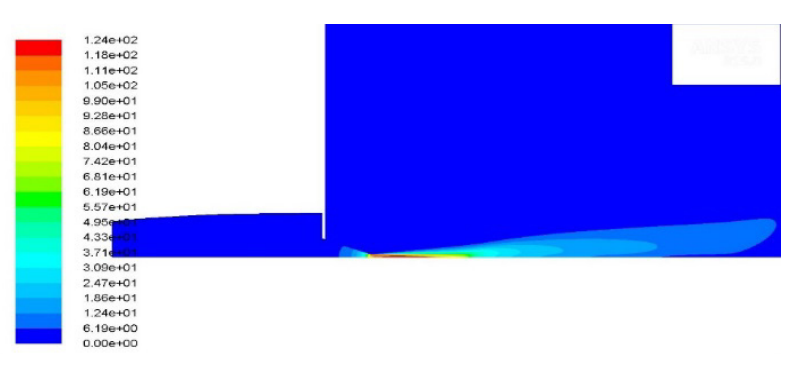

Figure 14d. Velocity magnitude $t=0.6 \mathrm{~s}$ 


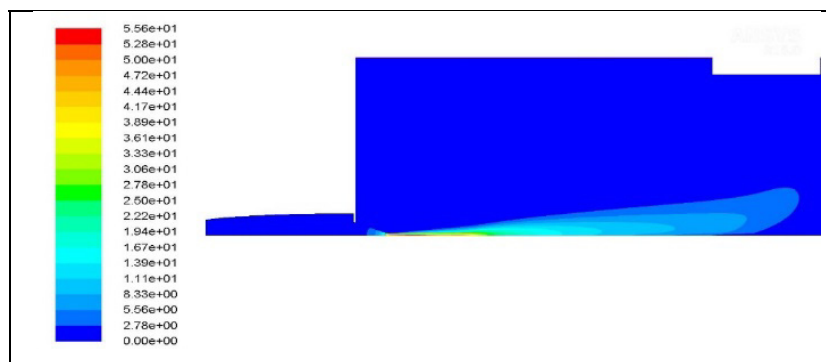

Figure 14e. Velocity magnitude $t=0.8 \mathrm{~s}$

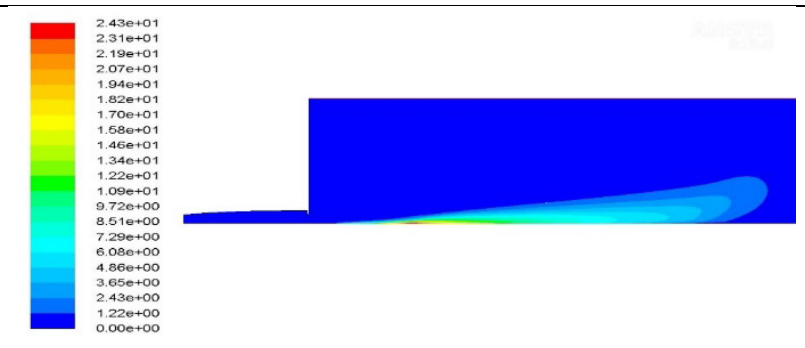

Figure 14f. Velocity magnitude $t=1$ s

The hydraulic impulse [16] of jet flow that was created by fluid movement is shown in Figure 15. It is noted that highest impulse is achieved when squid ejects water at 0.25 seconds that refers to Reynolds number of $2,542,372$. When kinetic energy calculations are performed, it is also realized that highest energy is left to the flow field at Reynolds number of 2,542,372. Besides, when jet duration is increased to 0.5 seconds from 0.25 seconds, value of kinetic energy drops more than half figure 16 .

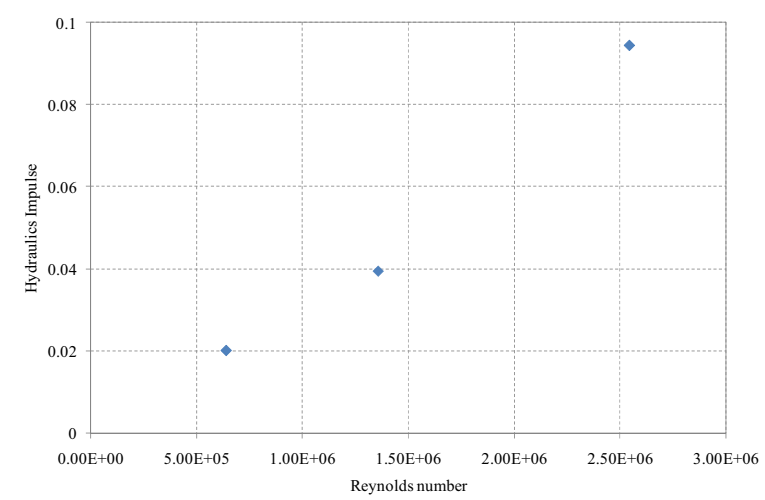

Figure 15. Impulse of jet flow.

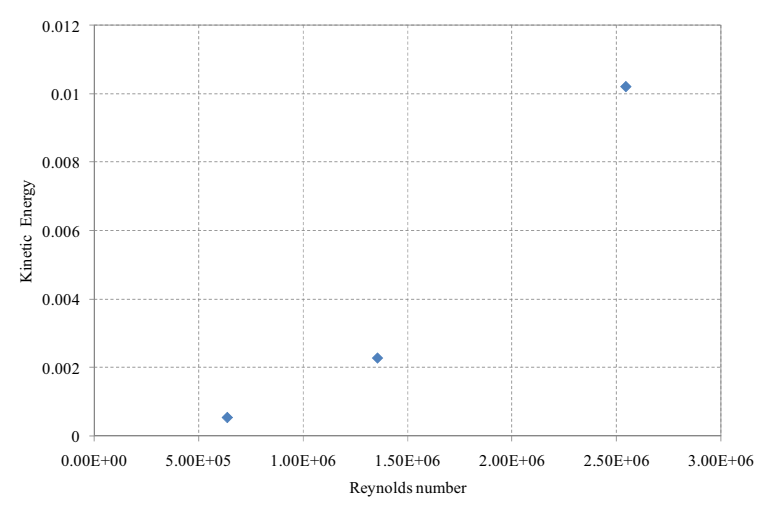

Figure 16. Kinetic energy of jet flow.

\section{Discussion and conclusion}

The construction of numerical models of biological geometries is an important contribution to the design of biomimetic structures and systems. In this study the construction of numerical model of a real squid using computed tomography scans is documented. The obtained model is successfully transferred to the analysis environment and the analysis studies have started. Wellstreamlined shape of the squid help to decrease drag coefficient at high Reynolds numbers, also help to delay separation. Squid propel themselves by using jet propulsion. Briefly, squid compresses the mantle cavity wall and pressurized water is ejected thru nozzle. This jet flow has a high momentum that generates locomotive force thus squid moves forward. The results that were obtained by numerical methods are in good agreement with experimental data of other marine animals.

\section{Acknowledgements}

This work has been supported by TUBITAK (The Scientific and Technological Research Council of Turkey) under 3501 Program, Project \#: 111M598.

\section{References}

1. F. E. Fish, Am. Zool. 36, 628-641(1996)

2. F. E. Fish, L. E. Howle, M. M. Murray, Integr. Comp. Biol. 48, 788-800 (2008)

3. L. L. Stelle, R. W. Blake, A. W. Trites, The Journal of Experimental Biology. 203, 1915-1923 (2000)

4. F. E. Fish, J. exp. Biol. 185, 179-193 (1993)

5. R. K. O’DOR, J. exp. Biol. 137, 421-442 (1988)

6. Md. M. Rahman, Y. Toda, H. Miki, Journal of Bionic Engineering. 8, 25-32 (2011)

7. L.Yu, C, S-C. Ting, Y.-H. Hsu, M-K. Yeh, J.-T. Yang, Mechanics Research Communications. 39, 59- 64 (2012)

8. XU. Yi-gang, W. De-cheng, Journal of Hydrodynamics 24(2), 263-272 (2012)

9. C. Eloy, Journal of Fluids and Structures. 30, 205218 (2012) 
10. G. Polidoria, Taiar, R. S. Fohannoa, T.H. Maia, A. Lodini, Journal of Biomechanics. 39, 25352541(2006)

11. A. Loebbecke, R. Mittal, F. Fish, R. Mark, Human Movement Science. 28, 99-112 (2009)

12. L. K. Bartol, P. S. Krueger, The Journal of Experimental Biology.Vol. 376212, 1889-1903 (2009)

13. E. J. Anderson, M. A. Grosenbaugh, The Journal of Experimental Biology. 208, 1125-1146 (2005)

14. M. Gharib, E. Rambod, K. Shariff, J. Fluid Mech. Vol. 360, 121-140 (1997)

15. M. Rosenfeld, E. Ramond, M. Gharib, J. Fluid Mech. Vol. 376, 297-318 (1998)

16. A. B. Olcay, P. S. Krueger, Theor. Comput. Fluid Dyn. (2009)

17. J. C. Bera, M. Michard, N. Grosjien, G. ComteBellot, Experiments in Fluids. 31,519-532 (2001)

18. X.U. Jing-lei, S. Jiang, L. Chun-feng, Kun-yuan, Zhang, Ser.B, 19, 453-458 (2007)

19. S. Carpy, R. Manceau, International Journal of Heat and Fluid Flow. 27, 756-767(2006)

20. K. Bremhorst, P. J. Gehrke, Experiments in Fluids. 28, 519-531(2000)

21. K.R. Kim, M.A. Cotton, T.J. Craft, O.R. Heynes, International Journal of Heat and Fluid Flow. 29 1650-1669 (2008)

22. M. Xiaoyong, G. Hongtao, F. Zhaolin, Z. Lin, Procedia Engineering. 31, 416 - 421(2012)

23. S. Hsua, Z. Travnicek, C.-Ch. Choua, C.-C. Chen, A.-B. Wang, Sensors and Actuators A. 203 (2013) 291- 299 (2013)

24. A. Yang, L. Jia, X. Yin, Journal of Bionic Engineering. 7, 103-108 (2010)

25. P. Cignoni, M. Callieri, M. Corsini, M. Dellepiane, F. Ganovelli, G. Ranzuglia, MeshLab: an OpenSource Mesh Processing Tool, Eurographics Italian Chapter Conference, V. Scarano, R. De Chiara, and U. Erra (Editors), 2-4 Temmuz 2008, Salerno, İtalya, 129-136 (2008)

26. Robert McNeal \& Associates, Rhinoceros NURBS modeling for Windows Versin 4.0 Users Guide, 113 s, A.B.D. (2008) 